\title{
Secondary researchers' duties to return incidental findings and individual research results: a partial-entrustment account
}

\author{
Henry S. Richardson, $\mathrm{PhD}^{1}$ and Mildred K. Cho, $\mathrm{PhD}^{2}$
}

Existing attempts to explain why secondary researchers might have any obligation to return findings to the contributors of genetic samples falter because of the lack of any direct interaction between the secondary researchers and the contributors. The partial-entrustment account of these obligations defended here circumvents this problem by explaining how a chain of special responsibilities can be forged even in the absence of any direct interaction.

Genet Med 2012:14(4):467-472

Key Words: ancillary care; biobanks; ethics; incidental findings; partial-entrustment model; returning research results
Collection of human biological specimens and data for research purposes is a common practice, one now central to many biomedical investigations. Perhaps equally widespread is the practice of conducting secondary research on biological samples and associated data ("samples," for short) that other researchers had collected for an earlier research project. How best to think about the ethics of such sample use by secondary researchers is still unclear, for several reasons. First, in the United States, some research with samples is currently considered "human subjects research" and regulated as such, whereas some is not; classification as human subjects research is primarily determined by how the samples were obtained (i.e., "through intervention or interaction with the individual," per 45 CFR 46) and whether private, identifiable information was obtained. In addition, research on samples from the dead is not considered human subjects research. Furthermore, there is not yet consensus on how specific the informed consent must be to allow secondary uses of samples. We set aside those cases in which samples are sometimes obtained in the course of clinical care (such as surgical biopsies for diagnosis of disease) or public health programs (such as blood spots for newborn screening programs) and then used in research projects. Further, we will here confine our attention to situations in which recontacting the contributors is possible because they are alive and because someone retains the ability to link them to the samples.

In one typical research scenario, an identifiable researcher (who we may call the sample collector, or Researcher A) has interacted with a human to collect samples and/or data and obtain informed consent. Researcher B may then wish to conduct a different research project with Researcher A's samples, obtaining them directly from Researcher A. Typically, Researcher B will not receive any information about the contributors' identities. However, Researcher A may retain information about their identities and be able to link them to the samples (the samples are coded). Alternatively, the identity of the research participants may have been permanently removed (the samples are anonymized).

In another scenario, Researcher A or a clinician collects the samples and deposits them, either anonymized or coded, in a biobank (see ref. 1, Figure 1). If the samples are coded, either Researcher A or the biobank might retain the code that links the samples to individual identities. Several specific characteristics of the scenarios may have practical and moral significance for the obligation to return results of secondary research to participants, especially the degree of de-identification of samples, the relationship that the collector of these has to the participants, and the nature of the secondary research.

In this article, we set out and explore a model of secondary researchers' obligations to return individual research results (IRRs) and incidental findings (IFs) to those who donated the genetic and other biological samples and/or associated data on which the secondary research is done. As we have indicated, we will use the term "samples" to refer both to samples and to associated data donated for research. "Secondary researchers" are, for present purposes, those who obtained access to the samples without entering into any direct relationship with those who contributed the samples, either by seeking the contributors' informed consent or otherwise. Instead, secondary researchers obtain access to the samples from others. Speaking of secondary researchers' obligations to return findings does not imply that they should enter into any direct relationship with the contributors. Just as one can return a library book by putting it in the post, so too one can return a finding by asking a biobank or other intermediary to recontact the contributor. An "IF" is a "finding concerning an individual research participant that has potential health or reproductive importance and is discovered in the course of conducting research but is beyond the aims of the study." An "IRR" is a finding concerning an individual

${ }^{1}$ Department of Philosophy, Georgetown University, Washington, DC, USA; ${ }^{2}$ Stanford Center for Biomedical Ethics, Stanford Department of Pediatrics, Stanford, California, USA. Correspondence: Henry S. Richardson (richardh@georgetown.edu) 
contributor that has potential health or reproductive importance and is discovered in the course of conducting research and is not beyond the aims of the study. ${ }^{2}$ "biobank" is a structured resource that holds human biological samples and/or data for the purpose of genetic or genomic research over time. By this definition, biobanks include archived data sets as well as repositories of biological samples and collections amassed by individual researchers, with or without linked phenotypic data or medical records.

\section{THE DIFFICULTY OF GROUNDING SECONDARY RESEARCHERS' OBLIGATIONS TO RETURN FINDINGS}

Because secondary researchers lack a direct relationship with the contributors, the moral grounds for any obligation for secondary researchers to return IRRs or IFs (together, "findings") are difficult to make out. ${ }^{3}$ The general issue of IFs had been widely neglected; but there is a growing consensus that "primary researchers" (here, researchers with a direct, informed consentbased relationship with participants) have an obligation, if certain conditions are met, to return IFs. ${ }^{2,4}$ For primary researchers, a general principle favoring the return of IRRs is widely accepted, although its exact contours are debated. ${ }^{5}$ Where, however, the "primary researchers" function solely as sample collectors, and hence are not generating significant results, this principle is not as firmly established. Both with regard to IFs and IRRs, then, the nascent consensus in favor of recognizing some obligation in the primary researchers to return these findings to the contributors is somewhat fragile. Because the moral basis for any such obligation incumbent on secondary researchers seems even weaker, we must examine it with care.

One important potential basis, equally supportive of primary and secondary researchers' obligations to return findings, is the general duty of rescue. ${ }^{6,7}$ The general thought behind the duty of rescue-however, it is precisely formulated-is that if one can rescue someone from a dire outcome without significantly inconveniencing oneself, then one ought to do so. Because warning someone is often quite easy, the duty to warn is often a special case of the duty of rescue. In itself, the duty of rescue is insensitive to any relationship that may exist between the rescuer and the person in peril: One's duty to rescue someone having a heart attack will be the same whether or not that person is a participant in one's study. Because the possibility of secondary research (research without a fresh informed consent relationship) largely depends on the research being done on coded samples, however, effecting any "warning" by returning findings will be more complicated and difficult than it would be for primary researchers. Because significant time may have elapsed since the samples were initially collected and the researchers may be in a different location than the contributors, recontact may be a logistical challenge. Especially given these difficulties, it seems unlikely that the duty of rescue could suffice to underwrite very many of the instances of an obligation to return findings from research with banked samples that are currently being defended. ${ }^{1,8}$ These recent recommendations suggest that obligations to return findings apply not only when this is an easy way to rescue a contributor from a dire outcome but also, far more generally, when the finding in question reveals a "substantial risk" of a "serious outcome" that is in some way "actionable." The duty of rescue, with its in-built proviso about not inconveniencing the agent, is too weak to support any such conclusion. If the grounds were limited to the duty of rescue, very few cases of returning findings from research with samples would be obligatory. Hardly any cases involving secondary researchers would be, due to the increased inconvenience involved in their effecting any returns (whether directly or indirectly).

Most other rationales offered for an obligation to return findings appeal to the existence of a relationship between the researchers and the contributors. This is explicit in the Maryland court case Grimes v. Kennedy Krieger Institute, in which the appeals court held that a "special relationship" between the researchers and the participants grounded potential claims." Without fully explaining the basis of this special relationship, the court described it as resulting from the informed consent process wherein recruited subjects agreed to a set of procedures for collecting and handling medical information about them. Two other rationales that have been mentioned involve more veiled references to such a relationship.

One is the brief appeal that Shalowitz and Miller ${ }^{10}$ make to the idea that respect for persons requires not treating them as mere means. Drawing inferences from this broad principle requires caution. To be sure, this general ideal of respect for persons has special standing and a special meaning in the context of medical research, where it comes to bear on the participant-researcher relationship. Indeed, outside of the context of some relationship or other it is hard to make concrete sense of this ideal of treating people respectfully as a distinctive source of moral obligation. (By contrast, convinced Kantians take this ideal to sum up the entirety of our obligations, but critics of Kant have long noted the difficulties he has with generating concrete conclusions from his a priori moral law.) If someone is not aware of your existence, it is a real challenge to treat him or her (in contrast to his or her corpse or his or her things) either respectfully or disrespectfully. Yet contributors will not even be aware of the existence of any particular secondary researchers.

A parallel point applies to any appeal to the ideal of reciprocity. ${ }^{11}$ Reciprocity-a feature of "exchange" among individuals ${ }^{12}$ typically thought of as involving an appropriate mutuality of benefit and burden-is manifestly a feature of a relationship. Although one might apply the idea of reciprocity to relationships of which one of the parties was unaware, doing so would require some serious explanation. And in any case, it appears that neither Illes et al. ${ }^{11}$ nor Knoppers and Chadwick ${ }^{12}$ think of the appeal to reciprocity as being wholly distinct from the appeal to respect for persons or as applying outside the context of a relationship. Illes et al. wrote that their defense of an obligation to return findings was "based on researcher obligations to respect subjects' autonomy and interests, demonstrating reciprocity when subjects agree to participate in studies by communicating a finding that may 
have a health impact" (p. 783, emphasis added). Similarly, Knoppers and Chadwick point to autonomy and respect for individual research participants as the rationale for researchers providing increased data security, privacy and confidentiality, as well as options for how samples are to be used. For the primary researchers to let the contributors know during the informed consent process that they will be reciprocating by returning significant findings is indeed a way for them to treat the contributors respectfully. This kind of personal interaction, however, is foreclosed to secondary researchers. Hence, most of the rationales hitherto offered for returning findings in cases that do not trigger the duty of rescue fail to apply to secondary researchers.

The idea of custodianship might offer some help, here. Several different custodial roles have been proposed for biobanks, including that of a "tissue trustee" whose responsibility is to de-identify tissues, control access to samples and data, and to assure that uses are appropriate. ${ }^{13}$ Others propose a stewardship model to minimize risk to contributors and assure informed consent to the donation process, ${ }^{14}$ balancing potential harms to them with benefits to "the common good." ${ }^{14,15}$ A third proposal embraces a legally inspired understanding on which research participants transfer property rights to a biobank, which holds them as a trustee. ${ }^{15,16}$ Although these various custodial models appear above all aimed at articulating or overseeing the ethical responsibilities of biobanks, they might well be developed in a way that allows us to derive obligations incumbent on secondary researchers.

But do biobanks really have special, custodial responsibilities? Although we do not mean to assume or endorse any particular custodial approach, the partial entrustment model that we will now set out as a way of supporting and explaining secondary researchers' obligations to return findings can be viewed as explaining why they do. Accordingly, the model fits well with the idea that biobanks play a custodial role. The partial entrustment model thus supports a certain understanding of the role-obligations of the custodians of samples and of secondary researchers. Rather than assuming that these people have such role-obligations, however, we seek to explain how the partial entrustment model grounds the claim that they do.

\section{AN ALTERNATIVE APPROACH: THE PARTIAL ENTRUSTMENT MODEL}

The partial entrustment model originated as a view about medical researchers' ancillary-care obligations-their obligations to provide or arrange for medical care that research participants need but that is not required in order to carry out their studies safely or soundly. ${ }^{17,18}$ The model naturally extends to the return of IFs, for it holds that the aspects of the participants' health that are entrusted to the researchers' care are those that come to light via the study procedures. ${ }^{4}$ What thus comes to light will either pertain to what is under study, and hence be covered by the ethical requirement to provide or arrange for standardof-care treatment-arguably part of what is required to carry out a study safely-or else be incidental or ancillary.

In being limited to those aspects of the participants' health that come to light via carrying out study procedures, the model limits the scope of the posited entrustment. It also limits the posited entrustment in another way, namely on the basis of the strength of a given claim for ancillary care. The factors on which the strength of such claims crucially depends are set out and illustrated in Table 1.

According to the model, if a given claim to ancillary care is within the scope of what has been entrusted to the researchers and if the claim is sufficiently strong, then the researchers have an obligation to honor that claim.

The various custodial models for biobanks propose that documents be drawn up according to which contributors explicitly entrust custody of their materials to the banks. Although spelling things out during the informed consent

Table 1 The strength factors and how they apply in a biobanking context

\begin{tabular}{|c|c|c|}
\hline Strength factor & Explanation of the factor & The factor illustrated in genomic biobanking \\
\hline Vulnerability & $\begin{array}{l}\text { How much difference would getting the information in } \\
\text { question make to the health or welfare of the participant? }\end{array}$ & $\begin{array}{l}\text { Research on rare but life-threatening conditions that research } \\
\text { participants might not be aware that they have and whose } \\
\text { danger could be averted, such as long QT syndrome, would } \\
\text { suggest a high degree of vulnerability. }\end{array}$ \\
\hline Dependence & $\begin{array}{l}\text { How dependent is the participant on the research team for } \\
\text { getting that care? }\end{array}$ & $\begin{array}{l}\text { Some diagnostic tests might be relatively new, and the tests } \\
\text { themselves or the expertise to interpret them may not be widely } \\
\text { available outside a research setting. }\end{array}$ \\
\hline Gratitude & $\begin{array}{l}\text { Do the researchers owe the participant any debt of gratitude (as } \\
\text { yet undischarged by the provision of a reciprocal benefit) for his } \\
\text { or her willingness to cooperate with their scientific enterprise by } \\
\text { undergoing procedures that are risky, painful, or inconvenient? }\end{array}$ & $\begin{array}{l}\text { In the case of typical biobank research, might the research } \\
\text { participant risk the possibility of discrimination or stigma merely } \\
\text { for having participated in a particular research project? }\end{array}$ \\
\hline
\end{tabular}


process would be a possible way to implement the partial entrustment model, this model does not require anything like this. According to this model, research participants entrust the relevant aspects of their health to the researchers, not because they think they are entrusting them and not because they trust the researchers to act in any particular way. As we know, what participants expect researchers to do for them is sometimes prone to the therapeutic misconception ${ }^{19}-$ specifically, to the form thereof that involves an "unreasonable appraisal of the nature or likelihood of medical benefit from participation in the study, due to a misperception of the nature of the research enterprise." ${ }^{20}$ Rather, the model holds that what matters is that, during the informed consent process, the participants have waived certain of their rights that the researchers not touch or probe their bodies or collect their medical histories. The model's core argument is this: Having gotten the participants to waive their rights against such access to private aspects of their bodies, the researchers obtain special responsibilities to look after the fundamental values that those rights normally protect. Various accounts of the point of these rights that protect the privacy of medical information and of the body might be given; but one morally important function of these rights is to safeguard vulnerable aspects of individuals' fragile capacities for autonomous choice. This being so, when researchers invite and accept waivers of these rights, they accrue some special responsibilities to look after this value. Although we are not addressing questions of legal liability in this article, this aspect of the informed consent transaction is the central moral basis of the special relationship between researchers and research participants that the court in Grimes appeared to be struggling to articulate.

These special responsibilities ground a special duty of ancillary care-a duty that, unlike the duty of rescue-holds specifically toward one's research subjects. ${ }^{21}$ Hence, researchers' ancillarycare obligations arise, not from any attitudes or beliefs peculiar to this or that researcher or participant, but rather from the nature of the informed consent process. If that process is properly conducted, then, in addition to informing the participants and giving them a chance to express their autonomous choices, researchers are also, necessarily, obtaining permission to access private information pertaining to the participants' bodies and their health. ${ }^{22}$ It is in accepting these special permissions that, the model argues, researchers take on special ancillary-care obligations.

This partial entrustment model applies readily to the case of secondary researchers working with samples for which reidentifying the contributor remains possible (as we indicated at the outset, we are confining our attention to such cases). The secondary researchers will have to have obtained permission to access the samples from those who hold them (whether primary researchers or a biobank). In our view, for this to be a permissible transaction pertaining to the range of cases we are considering, the contributors had to have given the sample collectors permission (if only blanket permission) to make the samples available to secondary researchers. This does not mean that this permission had to rise to the level of informed consent. As we have seen, it is controversial whether informed consent requirements apply to research with banked genetic (or other) samples; but the model's core argument applies however the contributors have waived their rights against access to the body or to privileged health information. The argument applies equally well whether they waive these rights informally, or during an informed consent process, or during a contractual process (proposed by Mitchell et al. ${ }^{23}$ ) wherein contributors set or accept terms pertaining to the use of the samples or data they provide. At the outset, we defined "secondary researchers" in a way that implies that they did not obtain new consents. Still, even if it would not count as "informed consent," permission for secondary research to be carried out with the samples needs to have been provided by the contributors, presumably as part of the initial informed consent process that authorized the collection of the samples. It is this permission that then gets transmitted from the sample collectors to the secondary researchers. If this is right, then what biobanks or other custodians are doing when they give specific secondary researchers permission to access samples is to pass along a permission that they initially obtained from the contributors. Because that permission for a potentially wider dissemination of the information about the contributors' health and bodies is a further aspect of the contributors' waivers of their privacy rights, it falls directly under the rationale for the partial entrustment model. Accordingly, when the samples' custodians pass along this permission, they also pass along the ancillary-care responsibilities that attach to it.

It is important that the term "privacy rights" not cause confusion, here. The rights that matter, according to the model, are rights that one's body not be probed or touched in certain ways and that one's medical history not be looked into-unless one gives one's permission. The de-identification of samples greatly enhances researchers' ability to keep any information derived from privileged access to people's bodies and health information confidential, which is an important moral concern, but does not fundamentally matter to the researchers' ancillary-care responsibilities. These are not grounded in informational privacy risks but in a special responsibility to deal, more broadly, with threats to the contributors that are revealed by exercising privileged access to their medical information. As long as it remains possible to return findings to the contributors-say, because the biobank holds the key-those responsibilities, which derive from the permissions of access to bodily information that the researchers have obtained, are practically relevant and in force.

The core argument of the partial entrustment model of researchers' ancillary-care obligations thus applies undiminished to the case of secondary researchers. Although it is true that providing a warning or other information can typically be covered by the duty of rescue, the logistical complications of returning findings from secondary research, coupled with the relatively wide range of findings that are thought to be important to return, suggest that a basis in the stronger, special ancillary-care obligation is not overkill, but rather a useful way 
of understanding secondary researchers' obligations to return results.

\section{IMPLICATIONS OF THE MODEL: APPLYING THE TEST OF STRENGTH}

The recommendations of Wolf et al. ${ }^{1,2}$ and others ${ }^{8}$ distinguish between results that researchers are obliged to return, based on the characteristics of the findings and their potential utility (such as seriousness of the relevant condition, strength of the association between the finding and potential harm, and actionability), and results that are permissible to return because of more distal effects (e.g., on children or on reproductive choices). As we have argued, the partial entrustment model generally supports and explains an obligation to return findings because research findings necessarily fall within the scope of the special obligation that arises from the permissions granted to the sample collector and passed along to the secondary researcher. Whether the model will agree with the more specific recommendations of these other authors about the thresholds to be set for the return of results will depend on how the factors affecting the strength of contributor's claims to return of findings are generally affected by the nature of secondary research. As detailed in Table 2, the expectable differences in the factors of engagement, gratitude, and cost each tend to suggest that secondary researchers' obligations toward contributors will be somewhat weaker than will primary researchers' obligations, other things being equal. To be sure, a situation creating very close relationships between the primary researchers and contributors, such as in long-term longitudinal studies, would lead to permissions that carry strong obligations to support the contributor's capacity for autonomous choice. In such a case, although the secondary researchers' level of engagement with the contributors remains zero, the engagement factor may nonetheless indirectly count as intensifying their obligation to return findings.

\section{THE SPECIAL CASE OF BIOBANK RESEARCH: IMPLICATIONS FOR PROCESS AND GOVERNANCE}

So far, our discussion has mainly abstracted from whether the secondary researchers obtain samples directly from the primary researchers or (as a possibly distinct case) from a biobank. The partial entrustment model of course implies that the entrustment responsibilities apply, in the first instance, to those who permissibly obtain the samples, independently of whether these responsibilities get passed on to others who make use of the samples. As an intermediary between the primary and secondary researcher, and because of the key functions it can play in the process of returning research findings, the biobank introduces some additional, special considerations into the application of the partial entrustment model. Depending on its policies, the biobank may have taken on a range of roles relevant to returning results that were formerly the purview of the primary researcher, including identifying and recontacting the relevant contributors and determining whether the process or content of returning findings is consistent with informed consent. Therefore, the biobank also assumes the responsibilities and obligations associated with those roles.

The partial entrustment model also suggests that biobanks should make explicit the transfer of responsibilities to both secondary and primary researchers that are conferred by the passing on of the privacy waiver. Specifically, the material transfer agreements that biobanks prepare should state that secondary researchers take on some ancillary-care obligations and are responsible for identifying research findings that might meet the threshold for reporting to contributors, as well as for being familiar with any constraints on such reporting that may have been agreed to through informed consent processes. In addition, when biobanks accept samples from primary researchers, the biobank should make explicit whether primary researchers retain obligations to re-identify and/or recontact contributors, or have transferred these obligations to the biobank, so that the implications of depositing samples are clear to primary researchers. Furthermore, because the relationship of the contributor to the research enterprise is initially through the primary researcher, and not at all through the secondary researcher, any results that are returned should not be directly returned by the secondary researcher. Indeed, secondary researchers may be unable to identify or otherwise prohibited from identifying the contributors in order to return results. Accordingly, our talk of secondary researchers' "obligations to return findings" should be understood as shorthand for their "obligations to do their part in an appropriate process of returning findings."

Table 2 The likely strength of primary and secondary researchers' obligations, compared

\begin{tabular}{|c|c|c|}
\hline Factor & Primary researchers & Secondary researchers \\
\hline Vulnerability & Full range, from strong to weak & Full range, from strong to weak \\
\hline Dependence & $\begin{array}{l}\text { Full range; especially strong if there is no other (CLIA certified) } \\
\text { source of the information }\end{array}$ & $\begin{array}{l}\text { Full range; it is possible that there is no other (CLIA certified) } \\
\text { source of the information }\end{array}$ \\
\hline Engagement & At least some interaction, perhaps extensive & $\begin{array}{l}\text { None (by definition); researchers may lack knowledge of } \\
\text { contributors' preferences }\end{array}$ \\
\hline Gratitude & $\begin{array}{l}\text { Baseline of risk, inconvenience, pain; possible contributor } \\
\text { involvement in organizing sample collection }\end{array}$ & $\begin{array}{l}\text { Some additional marginal increment of risk (loss of privacy; } \\
\text { informational risks) }\end{array}$ \\
\hline Cost & Baseline logistical costs of recontacting & $\begin{array}{l}\text { Additional logistical costs due to need for a mediating } \\
\text { infrastructure (cf. ref. 1) and to the lapse of time }\end{array}$ \\
\hline
\end{tabular}

CLIA, Clinical Laboratory Improvement Amendments. 


\section{CONCLUSION}

Although there is a developing consensus that secondary researchers have some obligation to return IRRs and IFs to sample contributors, most of the potential rationales for this position that have been mentioned in the literature apply only awkwardly to the case of secondary researchers, if at all. As we have explained, however, the partial entrustment model, originally developed to account for medical researchers' ancillary-care obligations, extends in a natural way to secondary researchers. It provides a clear grounding for secondary researchers' obligations to return results. These obligations attach to the special permissions to handle samples and associated data, permissions that get passed along from the sample collectors to the secondary researchers. In addition, the model provides reasons for expecting that these obligations of secondary researchers will be somewhat weaker than the obligations of primary researchers who encounter a finding of like seriousness and actionability.

\section{ACKNOWLEDGMENTS}

This work was supported by National Institutes of Health (NIH) grant 2R01HG003178 (S.M. Wolf, principal investigator) to H.S.R. and M.K.C. M.K.C. was also supported in part by $\mathrm{NIH}$ grants 5P50HG003389 and UL1 RR025744.

\section{DISCLOSURE}

The authors declare no conflict of interest.

\section{REFERENCES}

1. Wolf SM, et al. Managing incidental findings and research results in genomic research involving biobanks and archived datasets. Genet Med, 2012;14:361-384.

2. Wolf SM, Lawrenz FP, Nelson CA, et al. Managing incidental findings in human subjects research: analysis and recommendations. J Law Med Ethics 2008:36:219-48.

3. Cho MK. Understanding incidental findings in the context of genetics and genomics. J Law Med Ethics 2008;36:280-285.

4. Richardson HS. Incidental findings and ancillary-care obligations. J Law Med Ethics 2008;36:256-270.
5. Shalowitz DI, Miller FG. The search for clarity in communicating research results to study participants. J Med Ethics 2008;34:e17.

6. Hawkins JS. Justice and placebo controls. Soc Theory Pract 2006;32: 467-496.

7. Merritt MW, Taylor HA, Mullany LC. Ancillary care in community-based public health intervention research. Am J Public Health 2010;100:211-216.

8. Fabsitz RR, McGuire A, Sharp RR et al.; National Heart, Lung, and Blood Institute working group. Ethical and practical guidelines for reporting genetic research results to study participants: updated guidelines from a National Heart, Lung, and Blood Institute working group. Circ Cardiovasc Genet 2010;3:574-580.

9. Grimes v. Kennedy Krieger Institute, Court of Appeals of Maryland, September Term, 2000, Nos. 128 \& 129; 782 A.2d 807(2001).

10. Shalowitz DI, Miller FG. Disclosing individual results of clinical research: implications of respect for participants. JAMA 2005;294:737-740.

11. Illes J, Kirschen $M, E d w a r d s ~ E$, et al. Incidental findings in brain imaging research. Science 2006:311:783-784.

12. Knoppers BM, Chadwick R. Human genetic research: emerging trends in ethics. Nat Rev Genet 2005:6:75-79.

13. Merz J, Sankar P, Taube SE, Livolsi V. Use of human tissues in research: clarifying clinician and researcher roles and information flows. J Invest Med 1997;45:561-566.

14. Jeffers BR. Human biological materials in research: ethical issues and the role of stewardship in minimizing research risks. ANS Adv Nurs Sci 2001;24:32-46.

15. Ashburn TT, Wilson SK, Eisenstein BI. Human tissue research in the genomic era of medicine: balancing individual and societal interests. Arch Intern Med 2000;160:3377-3384

16. Winickoff $D E$, Winickoff $R N$. The charitable trust as a model for genomic biobanks. N Engl J Med 2003;349:1180-1184.

17. Belsky L, Richardson HS. Medical researchers' ancillary clinical care responsibilities. BMJ 2004;328:1494-1496

18. Richardson HS, Belsky L. The ancillary-care responsibilities of medical researchers: an ethical framework for thinking about the clinical care that researchers owe their subjects. Hastings Cent Rep 2004;34:25-33.

19. Appelbaum PS, Roth LH, Lidz CW, Benson P, Winslade W. False hopes and best data: consent to research and the therapeutic misconception. Hastings Cent Rep 1987;17:20-24.

20. Appelbaum PS, Lidz CW, Grisso T. Therapeutic misconception in clinical research: frequency and risk factors. IRB 2004;26:1-8.

21. Richardson H. Moral entanglements: ad hoc intimacies and ancillary duties of care. J Moral Philosophy, in press.

22. Manson N, O'Neill O. Rethinking the Informed Consent Process in Bioethics. Cambridge University Press: Cambridge, 2007.

23. Mitchell R, Conley JM, Davis AM, Cadigan RJ, Dobson A, Gladden R. Genomics. Genomics, biobanks, and the trade-secret model. Science 2011:332:309-310. 